\title{
THE VECTOR CALCULUS GAP: Mathematics $\neq$ Physics
}

\author{
Tevian Dray \\ Department of Mathematics, Oregon State University, Corvallis, OR 97331, USA \\ tevian@math.orst.edu \\ Corinne A. Manogue \\ Department of Physics, Oregon State University, Corvallis, OR 97331, USA \\ corinne@physics.orst.edu
}

(24 September 1998)

\begin{abstract}
We discuss some of the differences between the way mathematicians and physicists view vector calculus. These differences have led to a gap between the way this material is traditionally taught by mathematicians and the way physicists use it. We also give some suggestions for narrowing the gap.
\end{abstract}

Keywords: vector calculus; mathematics for scientists/engineers; geometric interpretation

\section{INTRODUCTION}

Each of us has taught vector calculus for many years: one of us (TD) as part of the second year calculus sequence in the mathematics department, and the other (CAM) as part of the third year physics course on mathematics methods. The latter course officially has the former as a prerequisite, although there is considerable overlap in the material covered. In fact, part of the reason the physics course exists is to teach physics majors the "right" way to use this material, the sense being that the mathematics department has failed to do so.

We are fortunate in having had an opportunity to compare notes frequently: we are longstanding collaborators in mathematical physics, and we are married to each other! Yet even so our conversations originally focused on what material was covered when, e.g., discussing which week was devoted to Stokes' Theorem, or whether to cover it before or after the Divergence Theorem. It literally took us years to discover that these theorems as taught by mathematicians bear little resemblance to the versions taught by physicists. Because it took us so long to realize this despite our anomalously close connection between the two departments, we believe that others may be unaware of the extent of this "vector calculus gap". It is the purpose of this paper to correct that deficiency by calling attention to this gap, and to offer suggestions for closing it.

However, this paper is not intended as a scholarly treatise comparing the various approaches. It contains only anecdotal evidence.

In what follows, we have stereotyped the two approaches we describe as "mathematics" and "physics"; this reflects our own training. We could equivalently have called these approaches "pure" and "applied", respectively, as the latter is also appropriate for engineers, particularly electrical engineers. While there will of course be local variations from these stereotypes, we are continually amazed at how accurate they are in classifying textbooks. 


\section{MATHEMATICS OR PHYSICS?}

Examine almost any mathematics text on vector calculus and one thing stands out: Vectors are triples of numbers, i.e. vectors are defined in terms of their Cartesian components with respect to a fixed choice of coordinates.

Examine almost any physics text which uses vector calculus and one thing stands out: vectors are arrows in space. Coordinates are put in afterwards - if at all. Square problems are handled in square coordinates, and round problems are handled in round coordinates.

The mathematics approach has its advantages: it is easy to visualize, and it provides a general framework for all problems in vector calculus. For example, when defining surface integrals, it is straightforward to develop the formula

$$
\overrightarrow{\boldsymbol{N}}=-\frac{\partial z}{\partial x} \overrightarrow{\boldsymbol{\imath}}-\frac{\partial z}{\partial y} \vec{\jmath}+\overrightarrow{\boldsymbol{k}}
$$

for the (upward) normal vector to a surface of the form $z=f(x, y)$, leading to the formula

$$
d S=\sqrt{1+\left(\frac{\partial z}{\partial x}\right)^{2}+\left(\frac{\partial z}{\partial y}\right)^{2}} d x d y
$$

for the surface element. This is beautiful mathematics. It works for any surface of the given form. For example, it makes it easy to handle the case of a paraboloid, which many problems in calculus textbooks are devoted to.

There is however a big problem with this for undergraduate physics:

The paraboloid simply does not arise in undergraduate physics!

The single most important difference between the two schools of thought is that the mathematician wants to attack the general case, whereas the (undergraduate) physicist only needs to master a few examples. In fact,

The only surfaces in all of undergraduate physics are the plane, the cylinder, and the sphere!

This is not due to a fear of the computational difficulties associated with general surfaces, which could be addressed using computer algebra systems. Rather, it is a reflection of a deep principle in physics: The world is symmetric. ${ }^{1}$ For example, the hydrogen atom is round; quantum mechanical orbits are described using spherical harmonics.

The physics approach has its advantages: the symmetry of a problem is directly exploited, usually resulting in tremendous simplification, and the underlying geometry is emphasized. For example, while the above formulas in Cartesian coordinates can easily be applied to (half of!) the sphere, it is much simpler to work in spherical coordinates. It is then obvious that

$$
\vec{n}=\hat{\boldsymbol{r}}
$$

and a simple construction of an infinitesimal rectangle on the sphere leads directly to

$$
d S=(r \sin \theta d \phi)(r d \theta)=r^{2} \sin \theta d \theta d \phi
$$

\footnotetext{
${ }^{1}$ There are important exceptions, but few at the elementary level.
} 
There is of course a big problem with this for the mathematician: It is not obvious how to do this for the paraboloid, let alone for general surfaces. However, crucial concepts can easily get lost in the complexity of general formulas, especially at the introductory level.

Consider for instance the cross product of two vector fields, which is of course perpendicular to both. But this is not terribly transparent from the construction of $\vec{N}$ as

$$
\overrightarrow{\boldsymbol{N}}=\frac{\partial \overrightarrow{\boldsymbol{r}}}{\partial x} \times \frac{\partial \overrightarrow{\boldsymbol{r}}}{\partial y}
$$

where

$$
\overrightarrow{\boldsymbol{r}}=x \overrightarrow{\boldsymbol{\imath}}+y \overrightarrow{\boldsymbol{\jmath}}+z \overrightarrow{\boldsymbol{k}}
$$

The crucial fact that the $\theta$ and $\phi$ derivatives of $\overrightarrow{\boldsymbol{r}}$ are tangent to the sphere, while true, requires a certain level of sophistication to understand intuitively. Ultimately, this formula for $\overrightarrow{\boldsymbol{N}}$ tends to become just something else to memorize. Contrast this with the analogous computation in a spherical basis, which is simply

$$
\overrightarrow{\boldsymbol{n}}=\hat{\boldsymbol{\theta}} \times \hat{\boldsymbol{\phi}}=\hat{\boldsymbol{r}}
$$

where $\overrightarrow{\boldsymbol{n}}=\overrightarrow{\boldsymbol{N}} /|\overrightarrow{\boldsymbol{N}}|$ and each basis vector is the unit vector in the corresponding direction. ${ }^{2}$

\section{DIV, GRAD, AND CURL}

The "physics" approach is to use a basis such as $\{\hat{\boldsymbol{r}}, \hat{\boldsymbol{\theta}}, \hat{\boldsymbol{\phi}}\}$ which is adapted to the symmetries of the problem. This usually leads to significant simplifications when computing the gradient, curl, and divergence of a vector field.

For example, the computation of the gradient of the function $q / \sqrt{x^{2}+y^{2}+z^{2}}$ is straightforward but somewhat messy; the divergence of the resulting vector field is even worse. Alternatively, using $r^{2}=x^{2}+y^{2}+z^{2}$ and the formulas for the gradient and divergence in spherical coordinates, it is easy to see that

$$
\vec{\nabla}\left(\frac{q}{r}\right)=\frac{\partial}{\partial r}\left(\frac{q}{r}\right) \hat{\boldsymbol{r}}=-\frac{q}{r^{2}} \hat{\boldsymbol{r}}
$$

(the electric field of a point charge at the origin), and that away from the origin

$$
\vec{\nabla} \cdot\left(\frac{q}{r^{2}} \hat{\boldsymbol{r}}\right)=\frac{1}{r^{2}} \frac{\partial}{\partial r}\left(r^{2} \frac{q}{r^{2}}\right)=0
$$

(differential form of Gauss' law).

Similarly, finding $\vec{\nabla} \times \overrightarrow{\boldsymbol{F}}$ for

$$
\overrightarrow{\boldsymbol{F}}=\frac{-y \overrightarrow{\boldsymbol{\imath}}+x \overrightarrow{\boldsymbol{\jmath}}}{x^{2}+y^{2}+z^{2}}
$$

\footnotetext{
${ }^{2}$ Admittedly, the former computation helps to determine the surface element of the sphere, while the latter does not. But, as already pointed out, the surface element of a sphere is easy to find directly using the much more intuitive approach above. Alternatively, one can calculate directly from the infinitesimal rectangle above that $\overrightarrow{\boldsymbol{\sigma}}=\overrightarrow{\boldsymbol{n}} d S=(r d \theta) \hat{\boldsymbol{\theta}} \times(r \sin \theta d \phi) \hat{\boldsymbol{\phi}}=\left(r^{2} \sin \theta d \theta d \phi\right) \hat{\boldsymbol{r}}$.
} 
is quite messy in Cartesian coordinates, whereas in spherical coordinates we have

$$
\overrightarrow{\boldsymbol{F}}=\frac{\sin \theta}{r} \hat{\boldsymbol{\phi}}
$$

and, after looking up the formula for the curl in spherical coordinates (which is found on the inside front cover of many physics texts!), we obtain

$$
\vec{\nabla} \times \overrightarrow{\boldsymbol{F}}=\frac{1}{r \sin \theta} \frac{\partial}{\partial \theta}\left(\frac{\sin ^{2} \theta}{r}\right) \hat{\boldsymbol{r}}-\frac{1}{r} \frac{\partial}{\partial r}\left(\frac{r \sin \theta}{r}\right) \hat{\boldsymbol{\theta}}=\frac{2 \cos \theta}{r^{2}} \hat{\boldsymbol{r}}
$$

Furthermore, noting that the (outward) unit normal vector to the sphere is just $\overrightarrow{\boldsymbol{n}}=\hat{\boldsymbol{r}}$, it is now simple to compute the flux of the curl of $\overrightarrow{\boldsymbol{F}}$ over (part of) the sphere, i.e. to calculate

$$
\iint_{S}(\vec{\nabla} \times \overrightarrow{\boldsymbol{F}}) \cdot \overrightarrow{\boldsymbol{n}} d S
$$

whereas to do this in Cartesian coordinates (try it!) is a mess. ${ }^{3}$

Yes, the formulas for the gradient, curl, and divergence are somewhat messier here than in Cartesian coordinates, requiring either memorization or knowing where to look them up. ${ }^{4}$ We argue that this is a small price to pay given how few cases are needed in undergraduate physics. The increase in intuitive understanding is substantial: It is not immediately obvious to most students that the first (Cartesian) form of $\overrightarrow{\boldsymbol{F}}$ is tangent to the sphere, whereas this is clear from the second form.

\section{GEOMETRIC INTERPRETATION}

In the mathematics approach, div and curl are defined as differential operators on vector fields. It is then shown that they satisfy, respectively, the Divergence Theorem and Stokes' Theorem. If there is time (there often isn't), it is then pointed out that div has something to do with how much a vector field is diverging, and curl has something to do with how much it is rotating.

In the physics approach, this is turned around completely. For instance, Schey [1] defines div and curl in terms of, respectively, the Divergence Theorem and Stokes' Theorem. This amounts to defining these operators to measure the amount a vector field is, respectively, diverging and rotating. The actual content of the two theorems, then, is to derive the explicit coordinate expressions for div and curl from which the mathematics approach starts.

We acknowledge that this is a radical change in viewpoint compared to traditional mathematics. We emphasize it to make a point, namely that the geometric interpretation of div

\footnotetext{
${ }^{3}$ This example provides an excellent demonstration of the more subtle properties of Stokes' Theorem. Considered in the plane $z=0$, this is the classic example where Green's Theorem fails, since $\overrightarrow{\boldsymbol{F}}$ is not defined at the origin. Stokes' Theorem applied to the upper unit hemisphere avoids this problem, but leads to the given integral.

${ }^{4}$ It is of course possible to use the chain rule to convert from Cartesian coordinates as needed. But this defeats the whole purpose, which is to use the symmetry from the beginning. Schey [1] derives the necessary formulas directly from the geometric interpretation. A unified treatment of all orthogonal coordinate systems is in fact not much more difficult, and is given in most beginning texts in mathematical physics.
} 
and curl (and grad) deserves greater attention than it often gets. Conveying the ability to look at a vector field and say whether it has divergence and/or curl without calculating things should be a high priority for any course on vector calculus, regardless of approach.

\section{SUMMARY}

Both the mathematics and physics approaches have their advantages. As already stated, the mathematics approach adapts more readily to generic situations without much symmetry, whereas the physics approach excels in situations with lots of symmetry. We do not wish to see one approach replaced by the other. But the current situation is that the approaches tend to be taught without overlap by different departments; this "vector calculus gap" must be eliminated.

We do note with pleasure that some "reform" calculus texts do indeed emphasize the geometric interpretation; this is a step in the right direction. We would add to this a treatment of elementary orthogonal bases, specifically those adapted to spherical and cylindrical coordinates.

In any case, we strongly advocate that any introductory course in vector calculus clearly indicate the existence of both approaches, and the types of problems each is suitable to. How much detail in each case should depend on local circumstances, but this will typically require incorporating supplemental material which is not in existing textbooks.

\section{SUGGESTIONS FOR CHANGE}

Here are some ideas on how to bridge the "vector calculus gap". We are pleased to acknowledge that most, if not all, of these ideas are already being tried by somebody, somewhere. Try them yourselves.

\subsection{For Mathematicians}

- Talk to colleagues in other departments

Make a point of discussing the content of your vector calculus course with members of the physics and engineering faculty. Talk to people who actually teach the course, not committee heads or advisors. Make sure to discuss details, not just categories. Ask them to provide examples. Find out not only exactly when they need students to know each topic, but exactly how that topic will be applied. Coordinate your class to these constraints as much as possible.

- Use a different text

You probably didn't choose your calculus text because of the quality of its treatment of vector calculus. Use a supplemental text which emphasizes a different approach. A relatively inexpensive example is Schey [1]. 


\section{- Use a right-handed basis}

We are quite frankly shocked at the number of calculus texts which use a left-handed orientation for their choice of spherical coordinates. Sure, this is just a convention, and doesn't affect much in a calculus course. But mathematicians should use the same convention everybody else does.

To be explicit, the standard physics convention is $(r, \theta, \phi)$ where $\theta$ is the angle from the $z$-axis and $\phi$ is the angle in the $x y$-plane. ${ }^{5}$ The labeling of the radial coordinate is not important, but it is unfortunate to have conventions which reverse the roles of $\theta$ and $\phi$. In any case, it is crucial that the angles be given in the (geometrical, not alphabetical!) order given.

- Emphasize geometry, not algebra

Vectors are arrows in space, not (merely) elements of $\mathbb{R}^{3}$. Div, grad, and curl all have geometric interpretations, which do not depend on their coordinate representations. Emphasize this.

- Get outside speakers

Invite people from other departments to give colloquia. Emphasize the need to be able to understand each other. Consider having some of these people give guest lectures in the classroom.

\subsection{For Physicists and Others}

- Talk to colleagues in the mathematics department

Make sure they know what you need. See above.

- Design your own course

Prepare a syllabus for the material your students need. Use it as a basis for discussion. If that doesn't work, the surest way to make mathematicians realize your needs are not being met is to to teach your own version of the course instead.

\subsection{For Everybody}

- Merge existing courses

Consider developing a single course which teaches both vector calculus and electromagnetism. The course should be developed by (at least) both mathematicians and physicists, and jointly taught by these (and possibly other) departments.

\footnotetext{
${ }^{5}$ This of course conflicts with the use of $\theta$ in polar coordinates. We would recover that elegance by using $\phi$ for polar coordinates, but that is unlikely to be popular.
} 


\section{ACKNOWLEDGMENTS}

We are grateful to Tom Dick for his constant encouragement. We thank the referees for their detailed constructive criticism, and especially for suggesting several of the items in Section 6. Tevian Dray gratefully acknowledges an L L Stewart Faculty Development Award from Oregon State University, which supported the preparation of a Study Guide for vector calculus [2]. Corinne Manogue was supported in part by NSF Grant DUE-9653250; the opinions expressed are those of the authors, and not necessarily those of the Foundation.

\section{References}

[1] Schey, H. M. 1992. Div, Grad, Curl, and All That, 2nd edition. New York: W. W. Norton.

[2] Dray, Tevian. 1996 \& 1997. Calculus Study Guide, MTH 255 (Vector Calculus II). Corvallis, OR: Oregon State University.

\section{BIOGRAPHICAL SKETCHES}

Tevian Dray received his BS in Mathematics from MIT in 1976, his PhD in Mathematics from Berkeley in 1981, spent several years as a physics postdoc, and is now Professor of Mathematics at Oregon State University. He considers himself a mathematician, but isn't sure. (Neither is his department.) Most of his research has involved general relativity.

Corinne Manogue received her AB in Mathematics and Physics from Mt Holyoke in 1977, her PhD in Physics from the University of Texas in 1984, and is now Associate Professor of Physics at Oregon State University. She continues to be amazed to find herself a physicist. Most of her research has been related to quantum gravity and superstring theory. She is also the Principal Investigator for a major NSF-supported curriculum reform effort in upperdivision physics.

Corinne and Tevian have collaborated on many projects, including 2 children. They are currently trying to give a unified description of the fundamental particles of nature in terms of the octonions.

(To appear in PRIMUS: Problems, Resources, and Issues in Mathematics Undergraduate Studies.) 